\title{
Photovoltage loss in semi-transparent organic photovoltaic devices
}

Yuxin Xia, Xiaofeng Xu and Olle Inganäs

The self-archived postprint version of this journal article is available at Linköping University Institutional Repository (DiVA):

http://urn.kb.se/resolve?urn=urn:nbn:se:liu:diva-160574

N.B.: When citing this work, cite the original publication.

Xia, Y., Xu, X., Inganäs, O., (2019), Photovoltage loss in semi-transparent organic photovoltaic devices, Organic electronics, 74, 37-40. https://doi.org/10.1016/j.orgel.2019.06.051

Original publication available at:

https://doi.org/10.1016/j.orgel.2019.06.051

Copyright: Elsevier

http://www.elsevier.com/

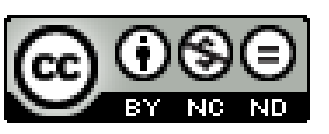




\title{
Photovoltage Loss in Semi-transparent Organic Photovoltaic Devices
}

Yuxin Xia, Xiaofeng Xu, Olle Inganäs*

Y. Xia, X. Xu, Prof. O. Inganäs, Biomolecular and Organic Electronics, Department of Physics, Chemistry and Biology (IFM), Linköping University, SE-581 83, Linköping, Sweden.

X. Xu, Department of Materials Science and Engineering, Ocean University of China , Qingdao 266100, China

E-mail: olle.inganas@liu.se

Keywords: Semi-transparent, Photovoltage loss, Photovoltaic devices

\begin{abstract}
The use of semi-transparent photovoltaic devices causes an inevitable loss of photocurrent, as light transmitted has not been absorbed. This trivial effect also leads to a loss of photovoltage, an effect partially due to the lower photocurrent but also due to the geometry of the semitransparent photovoltaic device. We here demonstrate and evaluate this photovoltage loss in semi-transparent organic photovoltaic devices, compared with non-transparent solar cells of the same material. Semi-transparent solar cells in addition introduce photovoltage loss when formed by lamination. We document and analyze these effects for a number of polymer blends in the form of bulk heterojunctions.
\end{abstract}

\section{Introduction}

Some of the main advantages of organic solar cells are those functions that established photovoltaics do not do that well; light weight, flexibility, semitransparency, design of colours. We have focused on semi-transparent devices on polymer substrates, in order to exploit these advantages. We are also focusing on polymer/polymer blends in the active photovoltaic layer; this suppresses some of the issues of unstable morphologies in polymer/molecular blends, which today are the highest performance materials in organic solar cells. Reported power conversion efficiency(PCE) in single junctions is now $14-16 \%,{ }^{[1-5]}$ and tandems have record of 
17.3\%. ${ }^{[6]}$ For the future application of OPV, it is essential that both efficiency and stability is combined. Polymer/polymer blends have shown great promise towards the stability goal. ${ }^{[7,8]}$ The still lower efficiencies may become better with more development; it is only 5 years ago that the long hibernation of polymer/polymer blends at 2-3\% PCE ended, and these materials are now demonstrated close to $10 \%$ in single junctions. ${ }^{[9-16]}$

The promise of scalable production at low capital cost due to printing of electrodes and active material on flexible polymer foil is a main driver for developing both materials and processing methods. The advantage of printing polymer/polymer blends comes from the higher viscosity of such solutions. We have further introduced lamination of active layer to active layer in devices as a construction method. This has great advantages in removing some of the problems of short circuits when printing multiple layers by sequential solutions deposition. Swelling of bottom electrode layers because of solvents introduced through defects in a top layer is a severe problem with fully printed solar cells. ${ }^{[17,18]}$

Our choice of geometry is the semi-transparent solar cells based on polymer/polymer blends sandwiched between semi-transparent electrodes of the ubiquitous PEDOT(PSS). One of these electrodes is modified with an interlayer to convert the electrode to be selective for electron extraction. Coating of a PEDOT(PSS) with polyethylene imine(PEI), or with ZnO nanoparticles, are standard methods of generating the cathode function. Construction of the photovoltaic cell starts by deposition of thin PEDOT(PSS) layers on PET foil. An interlayer is printed for the cathode function on one substrate. Both anode and cathodes are coated with an active layer by spin-coating, blade-coating or slot die coating. The two substrates are then laminated at a higher temperature and pressure, to make a mechanical and electrical connection between the surfaces. For organic photovoltaics, the performance has been limited by the relatively low photovoltage relative to absorption bandgap. Voltage losses are assigned to the relatively low efficiency of dissociation of photogenerated excitons and to the non-radiative charge recombination. Studies on photovoltage loss has related the loss to different parameters quantitatively by applying 
different methods. By applying detailed balanced theory, ${ }^{[19,20]}$ the photovoltage can be determined by simply measuring the photovoltaic external quantum efficiency $\left(\mathrm{EQE}_{\mathrm{PV}}\right)$ and electroluminescence external efficiency $\left(\mathrm{EQE}_{\mathrm{EL}}\right)$.

The photovoltage of these laminated devices is lower than that for the same material utilized in a standard geometry with a reflective top electrode, under standard illumination conditions. We evaluate the loss of photovoltage by measuring the external quantum yield of electroluminescence in different device configurations, and for several materials. We also measure the external quantum yield of photocurrent for the same devices. The lower photocurrent is a trivial consequence of the smaller optical absorption; around $30 \%-40 \%{ }^{[17]}$ of light is transmitted and can thus not contribute to charge carriers in the device, and thus leads to photovoltage loss. In this paper, we evaluate the radiative loss of $V_{\text {oc }}$ by the approximative treatment according to detailed balance theory. For the semi-transparent and laminated devices, we show that the loss of photovoltage with respect to the standard geometry is fully predicted by the sum of nonradiative and radiative voltage loss.

\section{Results and Discussion}

To identify the $V_{\text {oc }}$ loss of semitransparent devices in a general sense, three different all-polymer blend that have $V_{o c}$ varying from 0.81 to $0.89 \mathrm{~V}$ in normal structures were adapted into lamination method, and the comparison between the normal and laminated semitransparent devices was done. The polymer blends used in this paper include: TQ1:PNDI-T10 and PTB7Th:PNDI-T10 ${ }^{[17]}$, PBDTTS-FTAZ:PNDI-T10 ${ }^{[21]}$. In the following part, we will only use the donor name to represent the blend for convenience. Laminated devices are fabricated following reference 15. Compared to the normal structure device ITOLPEDOT:PSS(4083)\active layer $\backslash$ LiF\Al, different $V_{\text {oc }}$ loss was found, see Table 1. The smallest difference occurs for PTB7-Th devices, only $0.01 \mathrm{~V}$ and the largest is $0.09 \mathrm{~V}$ for TQ1. A very useful tool to analyze $V_{\mathrm{oc}}$ loss has been given by applying detailed balance theory on the solar cells, which separate 
the $V_{\mathrm{oc}}$ loss into two parts, the radiative and non-radiative, and quantify both using experimentally measurable parameters. Equation (1)-(3) shows how to calculate $V_{\mathrm{oc}}$ according to detailed balance theory, ${ }^{[19,20]}$

$V_{o c}^{r a d}=\frac{k_{B} T}{q} \ln \frac{J_{s c}}{J_{0}^{r a d}}$

$J_{0}^{r a d}=q \int_{0}^{\infty} E Q E_{P V}(E) \frac{2 F_{a}}{h^{3} c^{2}} \frac{E^{2}}{e^{\frac{E}{k_{B} T_{a}}}} d E$

$V_{o c}=V_{o c}^{r a d}+\frac{k_{B} T}{q} \ln E Q E_{E L}$

where $k_{\mathrm{B}}$ is Boltzmann constant, $\mathrm{T}$ is temperature, $\mathrm{h}$ is Planck constant, $\mathrm{c}$ is light speed, $\mathrm{T}_{\mathrm{a}}$ is the ambient temperature and $q$ is elementary charge. $V_{o c}^{r a d}$ is the radiative open circuit voltage, the upper limit of open circuit assuming all recombination is radiative. The term $\frac{k_{B} T}{q} \ln E Q E_{E L}$ is the non-radiative voltage loss. $E Q E_{P V}(E)$ is the external quantum efficiency, which is equal to emission possibility under equilibrium. The term $\frac{2 F_{a}}{h^{3} c^{2}} \frac{E^{2}}{e^{\frac{E}{k_{B} T_{a}}}}$ is the black body radiation. $J_{0}^{\text {rad }}$ is the emission current under equilibrium. $F_{\mathrm{a}}$ is a geometry factor, accounting for the light incidence or emission angle range. It would be $\pi$ for normal structure with reflective metal at back surface but should be $2 \pi$ for semitransparent structure with symmetric geometry.

Table 1. Comparison between laminated and normal structure devices in terms of difference of both radiative and non-radiative $V_{\mathrm{oc}}$ loss. The experimental $V_{\mathrm{oc}}$ are also listed and compared with prediction, showing minor error.

\begin{tabular}{|c|c|c|c|c|c|c|c|}
\hline & $\begin{array}{c}\text { EQE- } \\
\text { EL(\%) }\end{array}$ & $\begin{array}{l}\Delta V \text { non- } \\
\operatorname{rad}(\mathrm{V})\end{array}$ & $\begin{array}{c}\text { difference } \\
\text { of } \Delta \mathbf{V} \\
\text { non-rad } \\
\text { (V) }\end{array}$ & $\begin{array}{c}\text { difference } \\
\text { of } \Delta \text { Vrad } \\
\text { (V) }\end{array}$ & $\operatorname{Voc}(V)^{\text {a) }}$ & $\begin{array}{l}\text { Calculated } \\
\text { Voc } \\
\text { difference } \\
(V)\end{array}$ & $\begin{array}{c}\text { Experime } \\
\text { ntal Voc } \\
\text { difference } \\
\text { (V) }\end{array}$ \\
\hline TQ1 Normal & $3.81 \mathrm{E}-04$ & -0.324 & & & 0.91 & \multirow[t]{3}{*}{0.072} & \multirow[t]{3}{*}{0.09} \\
\hline \multirow[t]{2}{*}{ TQ1 Laminated } & $9.56 \mathrm{E}-05$ & -0.360 & & & 0.82 & & \\
\hline & & & 0.036 & 0.036 & & & \\
\hline PTB7-Th Normal & $9.17 \mathrm{E}-05$ & -0.359 & & & 0.80 & \multirow[t]{3}{*}{0.012} & \multirow[t]{3}{*}{0.01} \\
\hline PTB7-Th Laminated & $6.66 \mathrm{E}-05$ & -0.367 & & & 0.79 & & \\
\hline & & & 0.008 & 0.004 & & & \\
\hline
\end{tabular}




\begin{tabular}{ccccc|c|c}
\hline $\begin{array}{c}\text { PBDTTS-FTAZ } \\
\text { Normal }\end{array}$ & $7.39 \mathrm{E}-05$ & -0.364 & 0.87 & 0.033 & 0.03 \\
\cline { 1 - 1 } $\begin{array}{c}\text { PBDTTS-FTAZ } \\
\text { Laminated }\end{array}$ & $2.47 \mathrm{E}-05$ & -0.393 & & 0.84 & & \\
\hline
\end{tabular}

a) the measuring of Voc has an error of $\pm 0.005 \mathrm{~V}$.

The basic idea of detailed theory is that solar cells not only absorb sun light, but also exchange thermal photons with its surroundings. Under open circuit, the net absorption current and net emission current cancel each other. For devices without reflective back surface, the net emission gets larger, but the absorption does not, therefore the internal chemical potential, i.e. $V_{\mathrm{oc}}$, decreases in return to lower the emission. For a simple estimation at room temperature, assuming other parts of Equation 2 fixed, by changing $F_{\mathrm{a}}$ from $\pi$ to $2 \pi$ to account for the change from reflective to semitransparent, the loss of $0.026 * \ln (0.5) \approx 0.018 \mathrm{~V}$ is caused.

a)

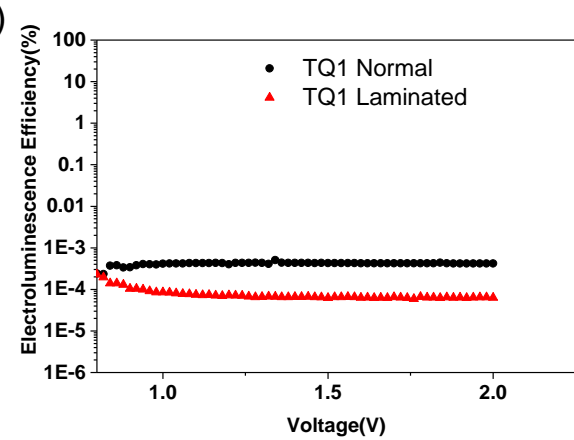

c)

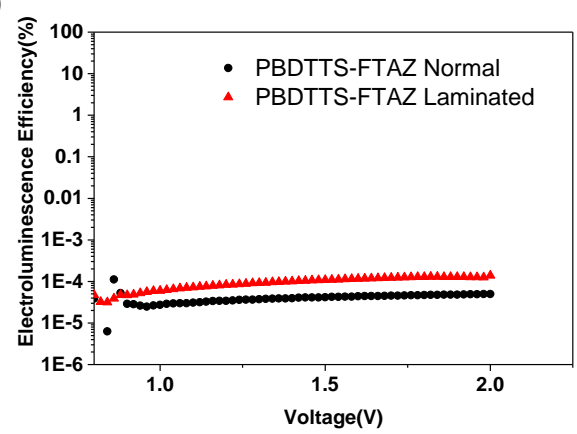

b)

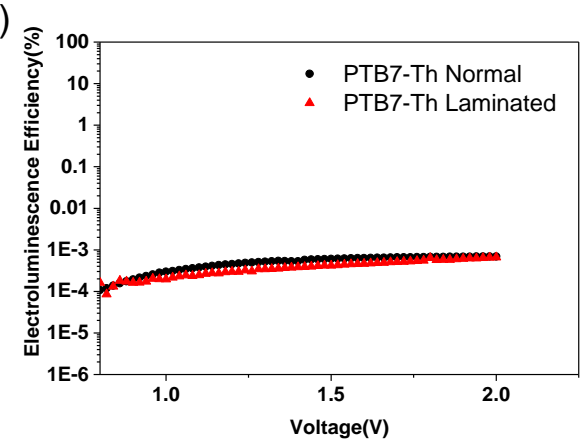

Figure 1. Electroluminescence efficiency of all normal and laminated devices. TQ1 and PBDTTS-FTAZ have difference and PTB7-Th shows little difference.

In Table 1, we list radiative and non-radiative loss of $V_{\text {oc }}$ separately for both laminated and normal devices. Electroluminescence (EL) efficiency and EQE were measured to calculate these losses. The EQEEL for PTB7-Th demonstrate little difference between laminated and 
normal devices but more different for TQ1 and PBDTTS-FTAZ, see Figure 1. As a result, the difference of $\Delta \mathrm{V}$ non-rad is the largest for TQ1, up to $0.036 \mathrm{~V}$ and $0.028 \mathrm{~V}$ for PBDTTS-FTAZ. The difference is only $0.008 \mathrm{~V}$ for PTB7-Th, which is quite small. As to the radiative loss, $V_{o c}^{r a d}$ is calculated using Equation 2 with measured EQE data demonstrated on Figure 2. The smallest difference also occurs for PTB7-Th, and the other two of $0.027 \mathrm{~V}$ and $0.036 \mathrm{~V}$ for PBDTTS-FTAZ and TQ1. As mentioned above, a 0.018V loss is expected due to the geometry change, but with certain assumptions. The assumption can be true when the EQE the same extent all over the absorption range, since the $J_{s c}$ and $J_{0}^{r a d}$ is just integration of $\mathrm{EQE} P \mathrm{PV}$ over different range. However, it does not hold when transferred to semitransparent devices with quite different preparation process. The morphology could be changed both during the spin-coating on a different substrate, and/or due to the lamination process with heat and pressure. For instance, absorption of thermally annealed TQ1 films gets redshifted. ${ }^{[17,22]}$ The latter process makes the shape of $\mathrm{EQE}_{\mathrm{PV}}$ change quite a bit. These influences can only be experimentally measured and would be superposed on the geometry loss to determine the total radiative loss.
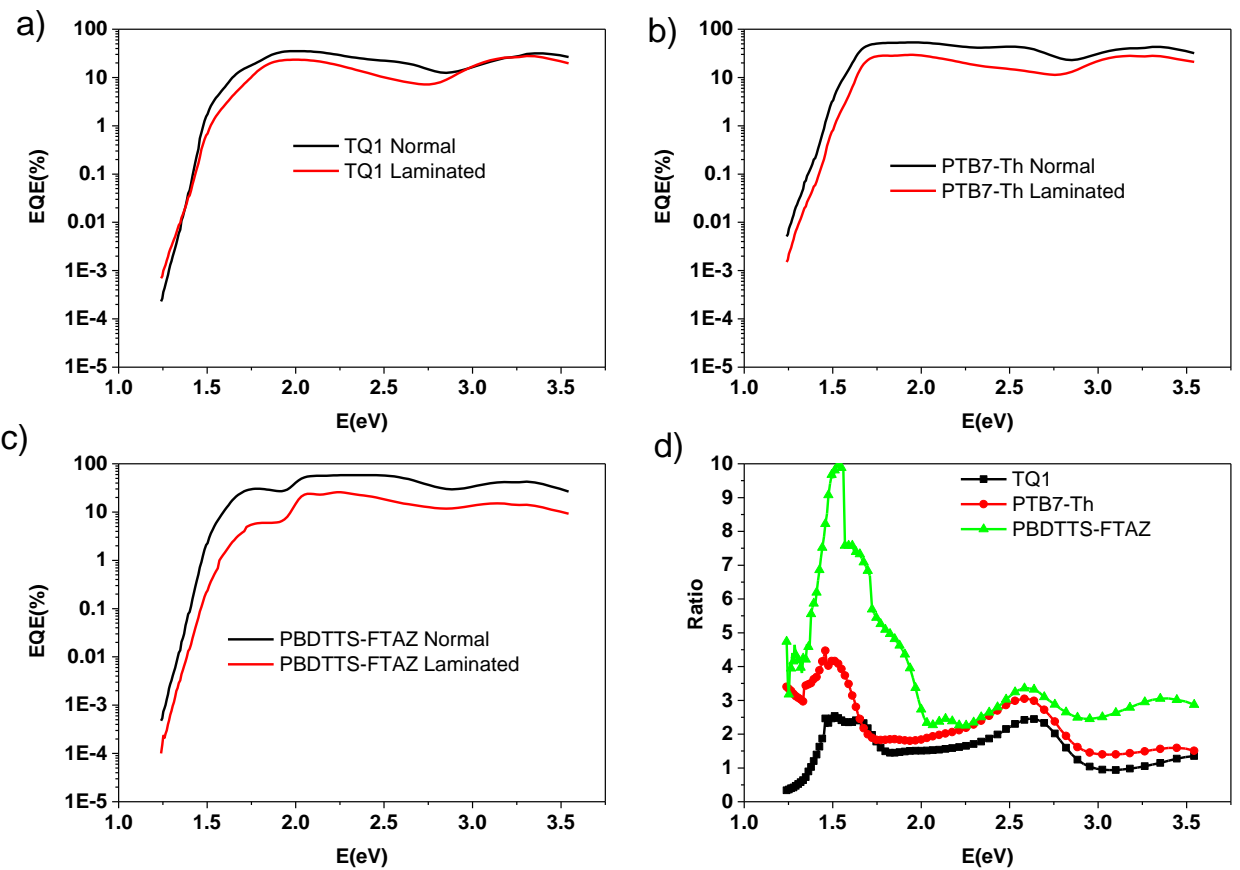

d)

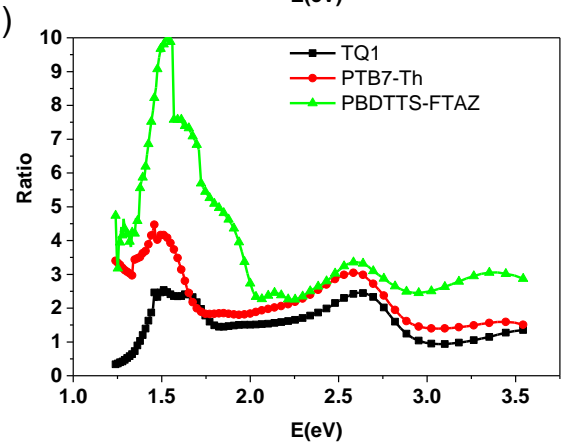


Figure 2. EQEPv of both normal and laminated devices a)-c). For TQ1, the EQE at lower energy range in laminated devices are comparable or even higher than the normal devices probably due to the morphology change. d) EQE ratio of normal devices over laminated devices.

Figure 2-d) shows the $\mathrm{EQE}_{\mathrm{PV}}$ ratio of normal over laminated devices. Apparently, the ratio at the lower energy range is very critical in the calculation of $J_{0}^{r a d}$, since the ambient radiation decrease exponentially with energy. This ratio for TQ1 is higher in the lower energy range $(<1.4 \mathrm{eV})$ than all other range, which means in the laminated devices the integration part in Equation 2 is getting smaller as well and leads to a further decrease of $V_{\text {rad. }}$ This results in a total loss of $V_{\text {rad }}$ larger than $0.018 \mathrm{~V}$. However, for both PTB7-Th and PBDTTS-FTAZ, we observe an opposite trend, the ratio is higher at lower range and will compensate the loss due to geometry, to make the total loss of $V_{\text {rad }}$ smaller than $0.018 \mathrm{~V}$, only $0.004 \mathrm{~V}$. Adding all the radiative and non-radiative loss together, we can then estimate the difference of $V_{\text {oc }}$ between laminated and normal devices. The results match well with experimental data with minor error. Even though the predicted difference is small, and might lie in experimental error bar, but it does not affect our main arguments here. It is also notable that, with more EQEPv data extended to even lower energy range, more precise prediction could be obtained.

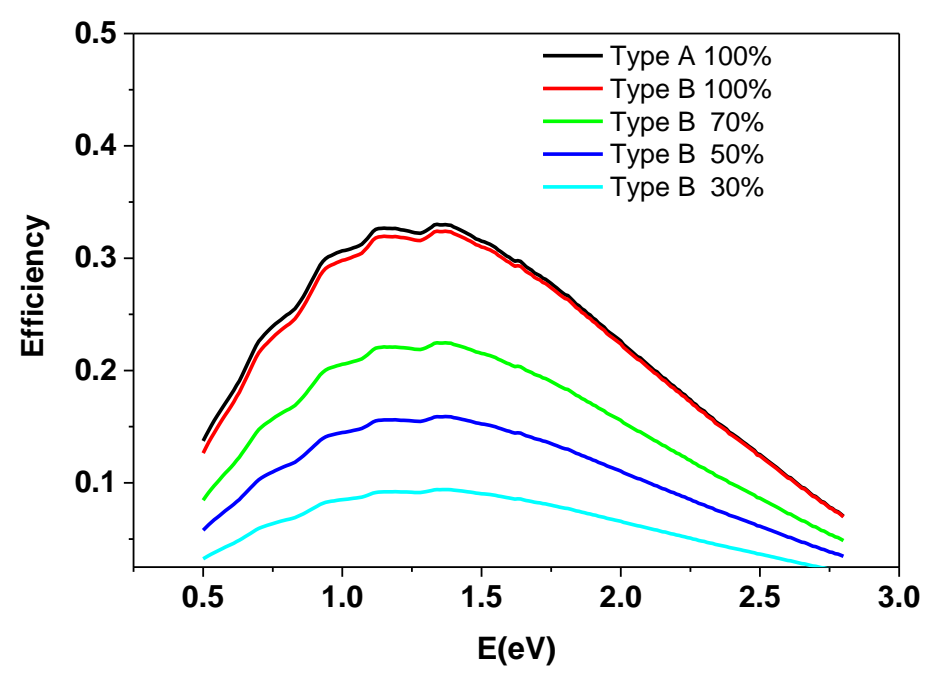

Figure 3 Prediction of PCE limit for devices with (Type A) and without (Type B) reflective back surface. Percentage indicate the different EQEPV. 
For devices without reflective back surface, both the photocurrent and photovoltage and thus power conversion efficiency is reduced. The theoretical upper limit to power conversion efficiency could be predicted using the detailed balance theory. In Figure 3, we show both normal devices with reflective surface(Type A) with $100 \% \mathrm{EQE}_{\mathrm{PV}}$, and devices without reflective surface (Type B) with $100 \%$ to $30 \% \mathrm{EQE}_{\mathrm{PV}}$ for photons with energy higher than bandgap. Doubling the parameter $F$ a will change the magnitude but barely the position of the highest efficiency along $\mathrm{X}$-axis. Without reflective surface the limit is a little bit decreased from $33 \%$ to $32 \%$. The limits for Type B indeed decrease in magnitude almost following the EQE number. It is noted that, for real devices, lamination might also change the $E_{\mathrm{g}}$ a little bit, and depending on different situations, PCE limit could be changed along with that change.

\section{Conclusion}

In this paper, we have demonstrated all polymer semitransparent organic solar cells using PEDOT(PSS) as electrodes on flexible PET foil. Lamination method was used for device fabrication. The fabrication has good promise of large-scale production of flexible and low cost organic solar cells. These advantages drive us to investigate photovoltaic properties of the laminated devices. By comparing them with normal structure devices with reflective back surface, in addition to the photocurrent loss due to semitransparency, there is photovoltage loss due to non-radiative photovoltage loss and another unavoidable $V_{\text {oc }}$ loss due to geometry. The EQE changes during fabrication of semitransparent devices by lamination, also play a role in the lower energy range. These different losses are analyzed using detailed balance theory. We designate the geometry $V_{\mathrm{oc}}$ loss as a radiative loss due to enhanced emission from doubled photon emission surface for semitransparent device. The difference of $V_{\mathrm{oc}}$ compared with the standard device is fully predicted by the model. We note that the semitransparent devices without any reflective back surface would always suffer a $V_{\text {oc }}$ loss of around $0.02 \mathrm{~V}$ because of the geometry. 


\section{Experimental section}

Device Fabrication and Characterization. PEDOT:PSS PH1000 (Heraeus) and PEI was printed according to the ref. 16. Active layers were spin-coated in glovebox separately on PH1000 and PEI modified PH1000, and then laminated inside glovebox using roll laminator with roll temperature $120^{\circ} \mathrm{C}$ and a force $\sim 55 \mathrm{~N}$ between two rollers. Force was measured by force sensor (FlexiForce A201, Tekscan). TQ1:PNDI-T10 (2:1 w\%) was spin-coated using solution with 12 $\mathrm{mg} / \mathrm{ml}$ concentration in xylene at $1000 \mathrm{rpm}$. PTB7-Th:PNDI-T10 (1:1 w\%) was spin-coated using solution with $12 \mathrm{mg} / \mathrm{ml}$ in $\mathrm{CB}$ at $1500 \mathrm{rpm}$. PBDTTS-FTAZ:T10 (2:1 w\%) was spincoated from CB with12mg/ml. To make BHJ devices, 4 nm PNDI-T10 was spin-coated at 3000 rpm using $4 \mathrm{mg} / \mathrm{ml}$ in xylene and then laminated with blend films on anode. PNDI-T10, PBDTTS-FTAZ and TQ1 was synthesized according to the literature. ${ }^{[9,23,24]}$ PTB7-Th was purchased from Solarmer Energy Inc, USA. J-V curves were recorded with Keithley 2400 Source Meter under illumination from a solar simulator (LSH-7320 LED Solar Simulator, Newport). EQE ${ }_{P V}$ was measured using Q-R Solar Cell Quantum Efficiency Measurement System (Enlitech). EL EQE was measured using a homebuilt system using a large-area calibrated Si photodiode from Oriel.

\section{Acknowledgements}

The work was funded by the Knut and Alice Wallenberg foundation (KAW), through a Wallenberg Scholar grant to O.I., and a Proof of Concept project for All Organic Printed Solar Cell Modules. Y. Xia acknowledges China Scholarship Council (CSC) for financial support. Notes

OI is co-founder of Epishine AB, developing laminated photovoltaic devices. 


\section{Reference}

[1] S. S. Li, L. Ye, W. C. Zhao, S. Q. Zhang, S. Mukherjee, H. Ade, J. H. Hou, Adv. Mater. 2016, 28, 9423.

[2] W. Zhao, S. Li, H. Yao, S. Zhang, Y. Zhang, B. Yang, J. Hou, J. Am. Chem. Soc. 2017, $139,7148$.

[3] S. Li, L. Ye, W. Zhao, H. Yan, B. Yang, D. Liu, W. Li, H. Ade, J. Hou, J. Am. Chem.

Soc. 2018, 140, 7159.

[4] J. Yuan, Y. Zhang, L. Zhou, G. Zhang, H.-L. Yip, T.-K. Lau, X. Lu, C. Zhu, H. Peng, P. A. Johnson, M. Leclerc, Y. Cao, J. Ulanski, Y. Li, Y. Zou, Joule 2019.

[5] B. Fan, D. Zhang, M. Li, W. Zhong, Z. Zeng, L. Ying, F. Huang, Y. Cao, Science China Chemistry 2019.

[6] L. Meng, Y. Zhang, X. Wan, C. Li, X. Zhang, Y. Wang, X. Ke, Z. Xiao, L. Ding, R. Xia, H.-L. Yip, Y. Cao, Y. Chen, Science 2018, 361, 1094.

[7] N. Balar, Y. Xiong, L. Ye, S. Li, D. Nevola, D. B. Dougherty, J. Hou, H. Ade, B. T. O'Connor, ACS Applied Materials and Interfaces 2017, 9, 43886.

[8] W. Kim, J. Choi, J.-H. Kim, T. Kim, C. Lee, S. Lee, M. Kim, B. J. Kim, T.-S. Kim, Chem. Mater. 2018, 30, 2102.

[9] Z. Li, X. Xu, W. Zhang, X. Meng, W. Ma, A. Yartsev, O. Inganäs, M. R. Andersson, R. A. J. Janssen, E. Wang, J. Am. Chem. Soc. 2016, 138, 10935.

[10] H. Yan, Z. Chen, Y. Zheng, C. Newman, J. R. Quinn, F. Dötz, M. Kastler, A. Facchetti, Nature 2009, 457, 679.

[11] L. Gao, Z.-G. Zhang, L. Xue, J. Min, J. Zhang, Z. Wei, Y. Li, Adv. Mater. 2016, 28, 1884.

[12] H. Kang, M. A. Uddin, C. Lee, K.-H. Kim, T. L. Nguyen, W. Lee, Y. Li, C. Wang, H. Y. Woo, B. J. Kim, J. Am. Chem. Soc. 2015, 137, 2359.

[13] B. Fan, L. Ying, Z. Wang, B. He, X.-F. Jiang, F. Huang, Y. Cao, Energy Environ. Sci 2017, 10, 1243.

[14] Z.-G. Zhang, Y. Yang, J. Yao, L. Xue, S. Chen, X. Li, W. Morrison, C. Yang, Y. Li, Angew. Chem. Int. Ed. 2017, 56, 13503.

[15] B. Fan, L. Ying, P. Zhu, F. Pan, F. Liu, J. Chen, F. Huang, Y. Cao, Adv. Mater. 2017, 29, 1703906.

[16] Z. Li, L. Ying, R. Xie, P. Zhu, N. Li, W. Zhong, F. Huang, Y. Cao, Nano Energy 2018, $51,434$.

[17] Y. Xia, X. Xu, L. E. Aguirre, O. Inganäs, J. Mater. Chem.A 2018, 6, 21186.

[18] J. Bergqvist, T. Österberg, A. Melianas, L. Ever Aguirre, Z. Tang, W. Cai, Z. Ma, M.

Kemerink, D. Gedefaw, M. R. Andersson, O. Inganäs, npj Flexible Electronics 2018, 2, 4.

[19] U. Rau, Phys. Rev. B 2007, 76, 085303.

[20] J. Nelson, The physics of solar cells, Imperial College Press, 2013.

[21] Z. Li, X. Xu, W. Zhang, X. Meng, Z. Genene, W. Ma, W. Mammo, A. Yartsev, M. R.

Andersson, R. A. J. Janssen, E. Wang, Energy Environ. Sci 2017, 10, 2212.

[22] Y. Xia, C. Musumeci, J. Bergqvist, W. Ma, F. Gao, Z. Tang, S. Bai, Y. Jin, C. Zhu, R.

Kroon, C. Wang, M. R. Andersson, L. Hou, O. Inganäs, E. Wang, J. Mater. Chem.A 2016, 4, 3835 .

[23] Z. Genene, J. Wang, X. Meng, W. Ma, X. Xu, R. Yang, W. Mammo, E. Wang, Advanced Electronic Materials 2016, 2, 1600084.

[24] E. Wang, L. Hou, Z. Wang, S. Hellström, F. Zhang, O. Inganäs, M. R. Andersson, Adv. Mater. 2010, 22, 5240. 